\title{
Oxidative resistance of leukemic stem cells and oxidative damage to hematopoietic stem cells under pro-oxidative therapy
}

\author{
Yongfeng Chen ${ }^{1}$, Yong Liang ${ }^{1}$, Xingjing Luo ${ }^{1}$ and Qiongying $\mathrm{Hu}^{1}$
}

\begin{abstract}
Leukemic stem cells (LSCs) and hematopoietic stem cells (HSCs) are both dependent on the hypoxic bone marrow (BM) microenvironment (also known as the BM niche). There is always fierce competition between the two types of cells, and the former exhibits a greater competitive advantage than the latter via multiple mechanisms. Under hypoxia, the dynamic balance between the generation and clearing of intracellular reactive oxygen species (ROS) is conducive to maintaining a quiescent state of cells. Quiescent LSCs can reside well in the BM niche, avoiding attack by chemotherapeutic agents, which is the cause of chemotherapeutic resistance and relapse in leukemia. HSCs acquire energy mainly through anaerobic glycolysis, whereas LSCs achieve energy metabolism largely through mitochondrial oxidative respiration. Mitochondria are the primary site of ROS generation. Thus, in theory, mitochondria-mediated respiration will cause an increase in ROS generation in LSCs and a higher intracellular oxidative stress level. The sensitivity of the cells to pro-oxidant drugs increases as well, which allows for the selective clearing of LSCs by prooxidative therapy. However, HSCs are also highly sensitive to changes in ROS levels, and the toxic effects of prooxidant drugs on HSCs poses a major challenge to pro-oxidative therapy in leukemia. Given the above facts, we reviewed studies on the oxidative resistance of LSCs and the oxidative damage to HSCs under pro-oxidative therapy. An in-depth investigation into the oxidative stress status and regulatory mechanisms of LSCs and HSCs in hypoxic environments will promote our understanding of the survival strategy employed by LSCS and the mechanism of the oxidative damage to HSCs in the BM niche, thus facilitating individualized treatment of leukemia patients and helping eliminate LSCs without disturbing normal hematopoietic cells.
\end{abstract}

\section{Facts}

1. Redox homeostasis is vital for maintaining the quiescence of LSCs. Quiescent LSCs can reside in the BM niche to avoid attack by chemotherapeutic agents, which is the cause of chemotherapeutic resistance and relapse in leukemia.

2. Theoretically speaking, LSCs undergoing mitochondria-mediated respiration will also exhibit an increased sensitivity to pro-oxidant drugs, which provides a basis for the leukemia treatments targeting redox homeostasis. However, the BM niche can protect LSCs from pro-oxidative treatments, and LSCs can also resist oxidative damage through antioxidative mechanisms.

3. For HSCs in hypoxic BM niches, a low ROS level is conducive to maintaining their stem cell features. A higher ROS level not only disrupts the quiescent state of HSCs but also may kill bone marrow hematopoietic stem cells (BMHSCs) or even cause BM suppression. It is then necessary to reduce the dosage or even completely stop chemotherapy.

Correspondence: Yongfeng Chen (cyfeng@tzc.edu.cn)

'Department of Basic Medical Sciences, Medical College of Taizhou University, Taizhou 318000 Zhejiang, China

Edited by Y. Shi

(c) (i) Open Access This article is licensed under a Creative Commons Attribution 4.0 International License, which permits use, sharing, adaptation, distribution and reproduction in any medium or format, as long as you give appropriate credit to the original author(s) and the source, provide a link to the Creative Commons license, and indicate if changes were made. The images or other third party material in this article are included in the article's Creative Commons license, unless indicated otherwise in a credit line to the material. If material is not included in the article's Creative Commons license and your intended use is not permitted by statutory regulation or exceeds the permitted use, you will need to obtain permission directly from the copyright holder. To view a copy of this license, visit http://creativecommons.org/licenses/by/4.0/. 


\section{Open questions}

1. In pro-oxidative treatment of leukemia, how can the protective effects of the BM niche on LSCs be blocked?

2. Leukemia is a disease of high heterogeneity, and the oxidative stress of leukemic cells varies across patients and dynamically within the same patient. A level of ROS that is too low during pro-oxidative treatment may be beneficial for the survival and proliferation of leukemic cells. However, if it is too high, it will exacerbate the damage to normal cells. Therefore, it is necessary to determine the optimal pro-oxidative treatment.

3. At present, studies on pro-oxidant therapy for leukemia are mainly conducted in animal experiments or in vitro cell experiments. Therefore, they cannot fully reflect the real situations in vivo. More convincing evidence is needed to reveal what the true redox state of LSCs and HSCs is in different types and stages of leukemia and whether there are significant differences in the sensitivity of the two cells to ROS.

4. The regulatory mechanism for redox homeostasis may differ between LSCs and HSCs. As such, is it possible to identify specific targets for pro-oxidative treatment to kill LSCs while avoiding damage to BMHSCs?

\section{Introduction}

Leukemia is a hematopoietic malignancy caused by mutations in BMHSCs or hematopoietic progenitor cells (HPCs). With the application of novel chemotherapeutic drugs and the progress in hematopoietic stem cells (HSCs) transplantation, the remission rate and diseasefree survival of leukemia patients have improved. However, during chemotherapy, leukemic stem cells (LSCs) may reside inside the $\mathrm{BM}$ niche in a quiescent state, evading the killing power of the chemotherapeutic agents. Thus, the protective effect of the BM niche on residual LSCs is the cause of chemotherapeutic resistance and relapse in leukemia ${ }^{1,2}$.

In a hypoxic $\mathrm{BM}$ niche, maintenance of quiescence and the biological functions of HSCs and LSCs, cell survival, and proliferation are closely related to the intracellular reactive oxygen species (ROS) level and oxidative stress status ${ }^{3}$. Much evidence in recent years has indicated that targeting the BM niche and disrupting redox homeostasis may be a new treatment strategy for leukemia ${ }^{4}$. However, HSCs are also highly sensitive to an increased ROS level. How to reduce the cytotoxic effects of ROS on HSCs while killing LSCs with a high ROS level represents another challenge in pro-oxidant therapy for leukemia. Therefore, an in- depth investigation into the oxidative stress status and regulatory mechanisms of HSCs and LSCs in hypoxic environments will promote our understanding of the survival strategy of LSCs in the BM niche and the limitations of HSCs in resisting oxidative injury. This understanding will help to develop individualized treatments that can protect normal BMHSCs while eradicating LSCs.

\section{Hypoxia is significant for maintaining the biological functions of HSCs}

HSCs in a hypoxic BM microenvironment mainly rely on anaerobic glycolysis for energy, and the ROS level associated with anaerobic glycolysis is relatively low. However, the differentiation of HSCs may lead to dynamic changes in the ROS level, and it was found that low endogenous ROS levels were crucial for maintaining the quiescence of $\mathrm{HSCs}^{5}$. Whereas an excessively high ROS level will drive HSCs to shift from the quiescent state, and their self-renewal capacity will be reduced, causing oxidative injury or even death of HSCs. Moreover, an increased production of ROS has also been associated with genomic instability and enhanced DNA damage, including double-strand breaks, and performs a signaling function to promote cell proliferation and migration, thus contributing to leukemic cell transformation ${ }^{6,7}$. It should be noted that ROS also has a pivotal role in innate immunity by acting as signaling molecules and as a direct effector that kills pathogens via phagocytosis. However, a persistently low ROS level in HSCs will not only lead to loss of stem cell function but also cause opportunistic infections ${ }^{8}$. Thus, the balance of ROS levels is critical for maintaining the biological functions of HSCs and host immunity.

In terms of the metabolism of HSCs, many regulatory mechanisms, including high antioxidant defense and a diversity of regulatory molecules, such as P53, FOXO3, Akt, MAPK, hypoxia inducible factor 1 (HIF-1) and ataxia telangiectasia mutated (ATM), are involved in maintaining the low ROS level of HSCs to protect HSCs from oxidative stress-induced injury ${ }^{9-13}$. Bone marrow stromal cells (BMSCs) residing in the HSC niche also has a vital role in maintaining the redox homeostasis of HSCs, and ROS in HSCs can be transferred to BMSCs, maintaining a low ROS level ${ }^{14}$. In addition, stromal cells can regulate the quiescence, proliferation, and differentiation of HSCs through direct contact with HSCs and secretion of a variety of cytokines via multiple signaling pathways (Fig. 1). Among them, the chemokine ligand 12 (CXCL12)/C-X-C chemokine receptor type 4 (CXCR4) axis has the most important role in the interaction between BMSCs and $\mathrm{HSCs}^{15}$. In addition, different types of immune cells and nerve cells are also involved in the regulation of the HSC microenvironment ${ }^{16,17}$. 
Quiescence HSC

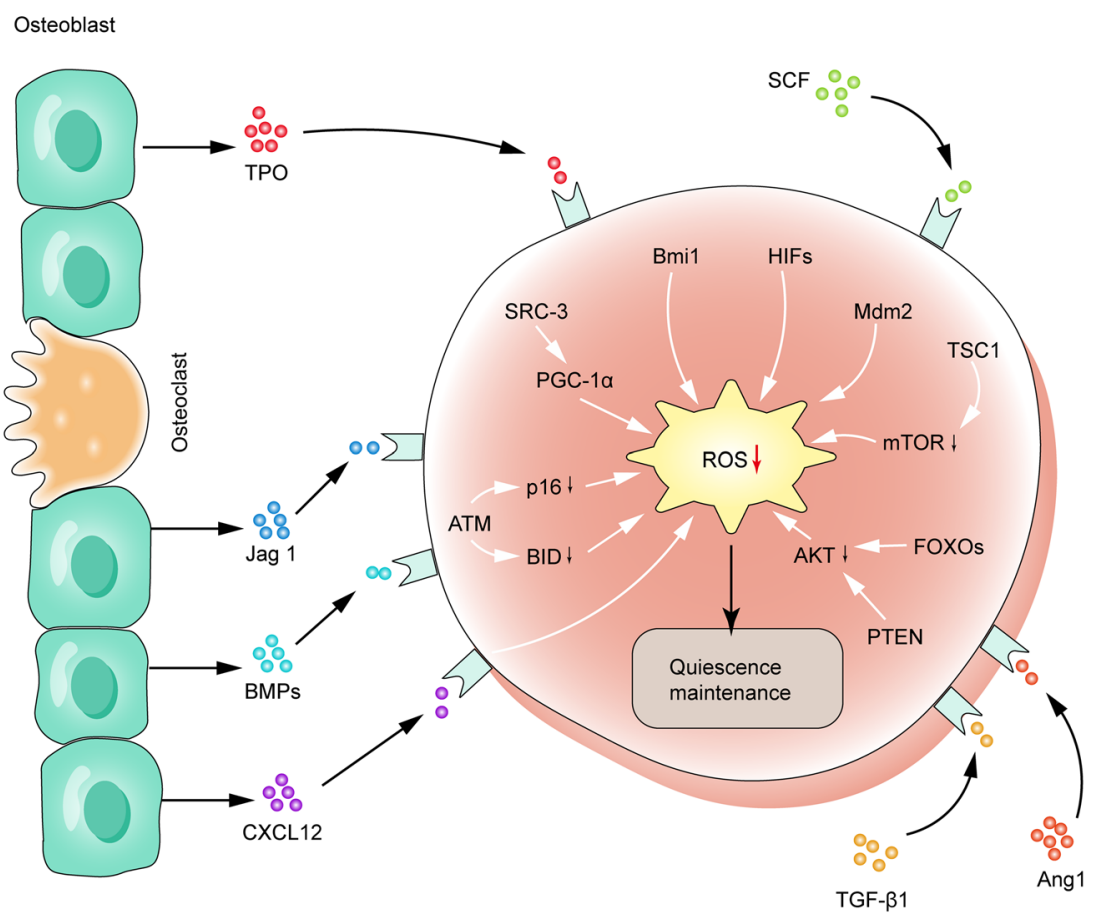

Fig. 1 Maintenance of redox homeostasis and quiescence of HSCs in the BM niche. The ROS level of HSCs in quiescence is regulated by a complex signaling network consisting of ATM, HIFs, FoxOs, SRC3, etc., which work together to maintain a low intracellular ROS level. The interaction between HSCs and BMSCs in the BM niche has a vital role in the long-term stability of HSCs. TPO, SCF, TGF- $\beta$ 1, and BMPs produced by BMSCs are all important regulators of the quiescence of HSCs.

LSCs are more adapted to the hypoxic environment of the BM niche than HSCs and inhibit the latter

LSCs and HSCs share similar self-renewal and differentiation features and other biological functions. For this reason, LSCs and HSCs are engaged in intense competition in the BM microenvironment, and the former usually have more advantages ${ }^{18,19}$. It has been shown that a hypoxic BM microenvironment can promote the synthesis of HIF-1a, which further induces the upregulation of CXCR4 on the surface of LSCs. The migration ability of LSCs can be enhanced through the interaction between CXCL12 and CXCR4, which facilitates the anchoring of leukemic cells in the BM niche and their quiescence. With the LSCs better protected in the BM niche than they are in circulation, their resistance to chemotherapeutic agents increases as well ${ }^{20-22}$.

As leukemic cells proliferate massively in the BM niche and secondary anemia occurs, hypoxia within the niche is exacerbated. Compared with normal HSCs, leukemic cells are more resistant to hypoxia. Goto et al. ${ }^{23}$ showed that leukemic cells can better survive in a hypoxic environment than HSCs by reducing ROS generation and enhancing ROS clearance. The leukemic cells can also release exosomes containing a variety of microRNAs, such as miR-210, which are then transported to the endothelial cells to inhibit the expression of the antiangiogenic factor EPH-related receptor tyrosine kinase ligand 3 (EFNA3), thus promoting angiogenesis ${ }^{24}$. In addition, HIF-1a ${ }^{25-28}$ and many cytokines, including granulocyte colonystimulating factor (G-CSF), granulocyte-macrophage colony-stimulating factor, CXCL12 and angiopoietin 1 (Ang1) secreted by BMSCs, are also involved in the regulation of angiogenesis ${ }^{29}$. The formation of more new vessels is conducive to the tolerance of the leukemic cells to the hypoxic environment and to the supply of oxygen and nutrients to LSCs for rapid growth. Recent reports indicate that HSCs acquire energy mainly through anaerobic glycolysis, whereas LSCs maintain energy metabolism and survival largely through mitochondrial oxidative respiration $^{30,31}$. According to recent studies, in human acute myeloid leukemia (AML) cells, the mitochondria of BMSCs can be transferred to AML cells via AML-derived tunneling nanotubes, a process that is dependent on the ROS generation mediated by nicotinamide adenine dinucleotide phosphate (NADPH) oxidases (NOX)dependent oxidative stress. Thus, more energy is supplied to AML cells through mitochondrial oxidative phosphorylation (Fig. 2). However, this phenomenon is not 


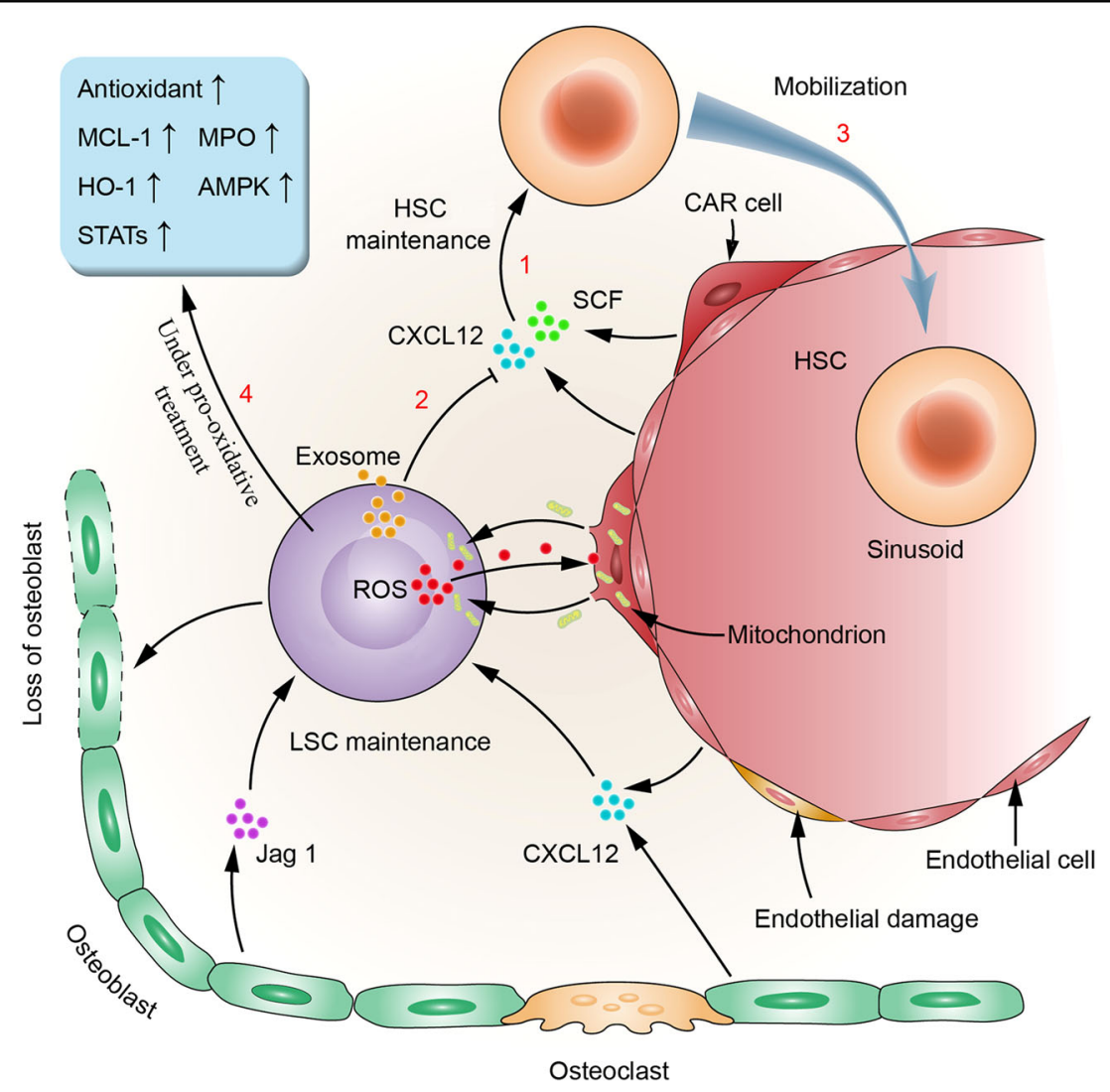

Fig. 2 LSCs' remodeling of the BM niche, inhibition of HSCs, and resistance against oxidative stress. LSCs have a remodeling effect of the BM niche by multiple pathways, such as activating the production of abnormal osteoblastic lineage cells from mesenchymal progenitor cells (MPCs). The interaction between LSCS and MSCS, including the transfer of ROS and mitochondria, is conducive to maintaining a low intracellular ROS level and energy metabolism of LSCs under a hypoxic environment. In addition, it has been indicated that 1. hematopoietic stem cell-supporting and retention factors secreted by bone marrow matrix cells, such as SCF and CXCL12 etc., have an important role in HSCs maintenance; 2. exosomes derived from leukemic cells may interfere and destroy HSCs maintenance by downregulating SCF and CXCL12; 3 HSCs mobilization in bone marrow niche is accelerated; 4. In the pro-oxidative treatment, LSCs can respond by upregulating antioxidant, MCL-1, MPO, and HO-1. As leukemia is a highly heterogeneous disease, the survival and redox regulation mechanisms of LSCs in the BM niche may vary for different types of leukemia. More studies are needed for revelation in this subject.

observed in $\mathrm{HSCs}^{32}$. Therefore, inhibiting angiogenesis and blocking the mitochondrial respiratory pathway of leukemic cells may help inhibit LSCs.

In addition to adaptation to the environment and competition for resources, leukemic cells can inhibit the number and activity of normal HSCs directly or by causing deterioration of the hematopoietic microenvironment. Kumar et al. showed that the expression of Dickkopf-related protein 1, which is a suppressor of normal osteogenesis and hematopoiesis, was elicited by AML-derived exosomes. Moreover, hematopoietic stem cell-supporting factors were downregulated in BM stromal cells, and their effect in supporting normal hematopoiesis was also decreased ${ }^{33}$. Huan et al. reported that critical retention factors (stem cell factor and CXCL12) were downregulated by AML-derived exosomes in stromal cells, and hematopoietic stem and progenitor cells were mobilized from the $\mathrm{BM}^{34}$. In addition, leukemic cells can induce remodeling of the BM niche by promoting the production of abnormal osteoblastic lineage cells from mesenchymal progenitor cell, thus transforming it into a normal hematopoiesis-suppressive and leukemia growth-permissive leukemia niche $\mathrm{e}^{35-38}$.

ROS levels are associated with the status of leukemic cells

It has long been known that increased production of ROS is a feature of tumor cells, and leukemic cells are not an exception, as they also exhibit elevated level of ROS. This feature is found in many leukemic cell lines and the cells of patients with various types of leukemia ${ }^{10}$. Compared with the levels in differentiated cancer cells, ROS levels are lower in cancer stem cells (CSCs), which is crucial for the survival of CSCs and the maintenance of their stemness. In contrast, an excessively high ROS level may trigger the death of $\mathrm{CSCs}^{39}$.

Recent studies have shown that similar to that of CSCs, the self-renewal ability of LSCs is also closely related to 
the ROS level and oxidative stress of the cells. According to Herault et al. ${ }^{40}$, LSCs highly expressed glutathione peroxidase 3 , and their ROS level was low, thereby maintaining the properties of stem cells. Lagadinou et al. analyzed ROS generation in primary AML cells, and it was found that AML cells with low ROS were more primitive than those with high ROS, exhibiting an immunophenotype and functional features of LSCs and largely being $\mathrm{G}_{0^{-}}$ stage, quiescent cells. This relatively dormant condition likely enables LSCs to persist, even under restrictive conditions such as low nutrient or oxygen levels ${ }^{31}$. LSCs account for $\sim 0.1-1.0 \%$ of leukemic cells in leukemia patients. Quiescent LSCs can reside in the BM niche to avoid attack by chemotherapeutic agents, which is the cause of chemotherapeutic resistance and relapse in leukemia. It has been shown that direct contact with BMSCs and regulation of the signaling molecules angiopoietin 1 (Ang1) and B-cell lymphoma 2 (Bcl-2) has important roles in maintaining the quiescence of LSCs ${ }^{31,41,42}$. Disrupting the quiescence of LSCs and inducing cell entry into the cell cycle in combination with administration of cell cycledependent chemotherapeutic agents can help eradicate the residual LSCs in clinical treatments for leukemia.

Compared with the level in more primitive leukemic cells in a quiescent state, the ROS level is higher in leukemic cells that are proliferating more ${ }^{30}$. Previous studies have shown that the mechanism of ROS generation in leukemic cells is very complex. When leukemic cells acquire energy through mitochondrial respiration, the mitochondrial respiratory chain become a very important source of intracellular $\operatorname{ROS}^{30}$. According to the literature, among various types of leukemia cells, including AML, chronic myeloid leukemia (CML) and promyelocytic leukemia cells, an increase in NOX activity is observed, indicating that NOX's constitutive activation is a very important source of intracellular ROS for $\mathrm{LSCs}^{43-45}$. Moreover, activated FMS-like tyrosine kinase and oncogenes such as $\mathrm{BCR} / \mathrm{ABL}$, cellular-myelocytomatosis viral oncogene and Ras are all closely related to changes in redox homeostasis in leukemic cells and increased ROS levels ${ }^{9,46,47}$. It has been found that antioxidant defense is decreased in different types of leukemia ${ }^{48-51}$, indicating that an imbalance between the oxidative and antioxidative systems may be one of the reasons for increased ROS levels in leukemic cells.

Recently, Bourgeais et al. ${ }^{52}$ found that the persistent activation of signal transducer and activator of transcription (STAT) 5 induced by BCR-ABL promoted ROS production in CML cells by inhibiting catalase (CAT) and glutaredoxin-1 (Glrx1) expression; however, when leukemic cells were cocultured with BM stromal cells to mimic a leukemic niche, CAT and Glrx1 were upregulated, causing downregulation of ROS levels and enhancement of leukemic cell quiescence. Given the facts above, antioxidant capacity may be related to the status of leukemia cells. It is believed that the downmodulation of some antioxidant systems contributes to the high level of ROS found in leukemic cells and that the upregulation of antioxidants allows the cells to survive under permanent oxidative stress without surpassing a deadly threshold. Furthermore, antioxidant upregulation promotes intracellular ROS elimination, maintaining cellular quiescence ${ }^{10}$.

\section{Targeting ROS in treatment for leukemia}

Mitochondria are the primary site of ROS generation. Thus, in theory, mitochondria-mediated respiration will cause an increase in ROS generation in leukemia cells and a higher intracellular oxidative stress level. The sensitivity of the cells to pro-oxidative drugs increases as well, which allows for the selective clearing of LSCs by pro-oxidative therapy ${ }^{53-57}$. The application of pro-oxidant chemotherapeutic agents may cause death of leukemia cells by increasing ROS, protein oxidation and mutation, lipid peroxidation, and mitochondrial stress and activating the $\mathrm{G}_{2} / \mathrm{M}$ phase cell cycle checkpoint ${ }^{10,58}$.

Studies have shown that a variety of chemotherapeutic agents for leukemia, including vincristine, doxorubicin, and cytosine arabinoside, work by promoting ROS generation $^{59-62}$. Constant optimization and the combined use of chemotherapeutic agents can help improve the outcomes of pro-oxidative treatment for leukemia ${ }^{63}$. Mitochondria are the main site for intracellular ROS generation. Therefore, targeting mitochondria is a reasonable strategy to disrupt the redox balance of the cells, induce oxidative stress and promote the apoptosis of leukemia cells ${ }^{64}$. A variety of mitochondrial inhibitors that can promote ROS generation are undergoing clinical trials for their role in leukemia treatment. Metformin, an antidiabetic drug, has been proven to be capable of inhibiting mitochondrial ATP generation and increasing ROS levels ${ }^{65}$. Adaphostine is another drug with proven ability to increase ROS levels by inhibiting mitochondrial respiration, this drug can overcome the resistance of primary CML cells to imatinib ${ }^{66,67}$. It should be noted that, a high ROS level not only induces cell apoptosis but also induces noncaspase-dependent necroptosis, which is conducive to overcoming the drug resistance mediated by the apoptotic defect ${ }^{68,69}$.

However, the leukemia niche can protect leukemic cells under oxidative stress. For example, BMSCs protect leukemic cells by activating prosurvival signaling pathways such as the PI3-K/Akt pathway ${ }^{70}$ and releasing protective molecules such as asparagine ${ }^{71}$, fatty acids $^{72}$, and cysteine $^{73}$. Leukemic cells can also relieve oxidative injury by interacting with the leukemia niche. Ding et al. ${ }^{74}$ reported that $\mathrm{H}_{2} \mathrm{O}_{2}$ generated by chronic lymphocytic leukemia (CLL) cells under vorinostat treatment was 
transferred to the surrounding stromal cells and drove autophagy, mitophagy, and glycolysis, resulting in the local production of high-energy mitochondrial fuels, which were then taken up by CLL cells to be effectively utilized through mitochondrial oxidative phosphorylation to enable more ATP production (Fig. 2). Under daunorubicin treatment, acute lymphoblastic leukemia (ALL) cells induce intracellular ROS production and oxidative stress responses in adipocytes, leading to the secretion of soluble factors that protect ALL cells from daunorubicin ${ }^{75}$.

Leukemic cells also have complex antioxidative mechanisms to resist oxidative stress. The intracellular antioxidant enzyme system consisting of superoxide dismutase (SOD), peroxidase, and CAT can clear away excessive ROS in cells, thus maintaining intracellular redox balance. Some small molecular substances, such as vitamins $E, C$, and $A$, can also clear away free radicals and prevent lipid peroxidation. In addition, the mercapto reductive buffer system consisting of glutathione (GSH) and thioredoxin (Trx) has a very important role in maintaining the intracellular redox state ${ }^{76}$. Several studies have demonstrated that suppression of the intracellular antioxidant system can help improve the efficacy of prooxidative therapy in leukemia ${ }^{77-81}$. It has been recently reported that nuclear factor erythroid 2-related factor 2 (Nrf2) is a key factor regulating the oxidative stress response of cells. Nrf2 is regulated by Kelch-like ECHassociated protein 1 (Keap1) and participates in the regulation of the activities of antioxidant enzymes such as SOD, CAT, and Trx by interacting with antioxidant response elements (AREs) ${ }^{82,83}$; Nrf2 can also promote the expression of various antioxidant proteins such as heme oxygenase enzyme- 1 (HO-1) and regulate the regeneration of $\mathrm{GSH}^{82,83}$. Suppressing the Nrf2 signaling pathway has been proven to effectively reverse the drug resistance of leukemia cells ${ }^{84}$. Besides, there are still many antioxidant molecules are involved in protecting leukemia cells from oxidative stress. Under the higher oxidative stress level caused by chemotherapy, ALL cells can upregulate antioxidant production and myeloid cell leukemia 1 expression ${ }^{85}$. Under cytarabine treatment, myeloperoxidase (MPO) expression is increased in AML cells, which promotes the conversion of hydrogen peroxide into hypochlorous acid, thereby reducing the sensitivity of AML cells to cytarabine ${ }^{86}$. In addition, a complex signaling network involving $\mathrm{HO}-1^{87}$, AMP-activated protein kinase $^{88}$, STAT $5^{89}$, and STAT $3^{90}$ also has an important role in maintaining redox homeostasis in leukemic cells (Fig. 2).

Recently, many studies have demonstrated that nitric oxide (NO), the main member of ROS, also exhibits antioxidation properties, which can destroy cascade reaction of lipid peroxidation and protect integrity of cytoplasm, avoiding oxidative injury of leukemic cells ${ }^{91,92}$.
However, NO also stimulates ROS production, thereby eliciting lipid peroxidation. Therefore, NO has dual roles in the body, which are dependent on its relative concentration in the body ${ }^{93,94}$. Moreover, much evidence shows that leukemic cells relieve oxidative stress via autophagy and clear the organelles damaged by oxidative stress. Thus, inhibiting autophagy is conducive to promoting the death of leukemic cells ${ }^{95-97}$.

Given the facts above, pro-oxidative treatment for leukemia requires an individualized strategy, and specific interventions should be based on the antioxidative features of different types of leukemic cells. Furthermore, blocking the microenvironment's protection of leukemic cells from antioxidants may contribute to better outcomes.

\section{Oxidative injury of HSCs caused by pro-oxidative treatment}

The killing power of ROS is not cell specific. Therefore, a high ROS level not only kills the leukemic cells but also undesirably causes oxidative injury or even death of HSCs. Tang et al. showed that the high ROS level induced by pro-oxidative chemotherapeutic agents not only caused direct injury and apoptosis of HSCs but also disrupted the $\mathrm{BM}$ niche where HSCs reside. This will ultimately damage the hematopoietic functions of the $\mathrm{BM}^{98}$. Continuous oxidative injury of DNA can also cause senescence and loss of the self-renewal ability of HSCs, which may be an important reason for long-term BM suppression and hematopoietic failure ${ }^{99,100}$. The proposed mechanism of HSC oxidative damage and senescence is illustrated in Fig. 3.

In addition, recent studies have demonstrated that autophagy serves as an important buffer system for oxidative stress and has a vital role in protecting HSCs from oxidative injury and maintaining the stem cell features of HSCs $^{101,102}$. However, autophagy is also a double-edged sword, and excessive autophagy will cause autophagic cell death $^{13}$. As it has been shown that ROS are an important factor inducing autophagy ${ }^{103}$, the autophagy level of LSCs, which depends on mitochondrial respiration, may be theoretically higher than that of HSCs, which depends on glycolysis ${ }^{104,105}$. However, this hypothesis remains to be further confirmed by more evidence. To date, targeting autophagy to eradicate LSCs via autophagic cell death without disturbing normal hematopoietic cells has aroused widespread attention in researchers ${ }^{106,107}$.

To address the adverse impact of pro-oxidative treatment on BM hematopoietic function, inhibition of leukemic cells' intracellular antioxidants, e.g., $\mathrm{GSH}^{108}$ and HO- $1^{109}$, may be an effective strategy. In theory, antioxidant inhibitors can also cause increased intracellular ROS levels, which means they can be used as prooxidants. Isothiocyanates is such a pro-oxidant drug ${ }^{110}$. 


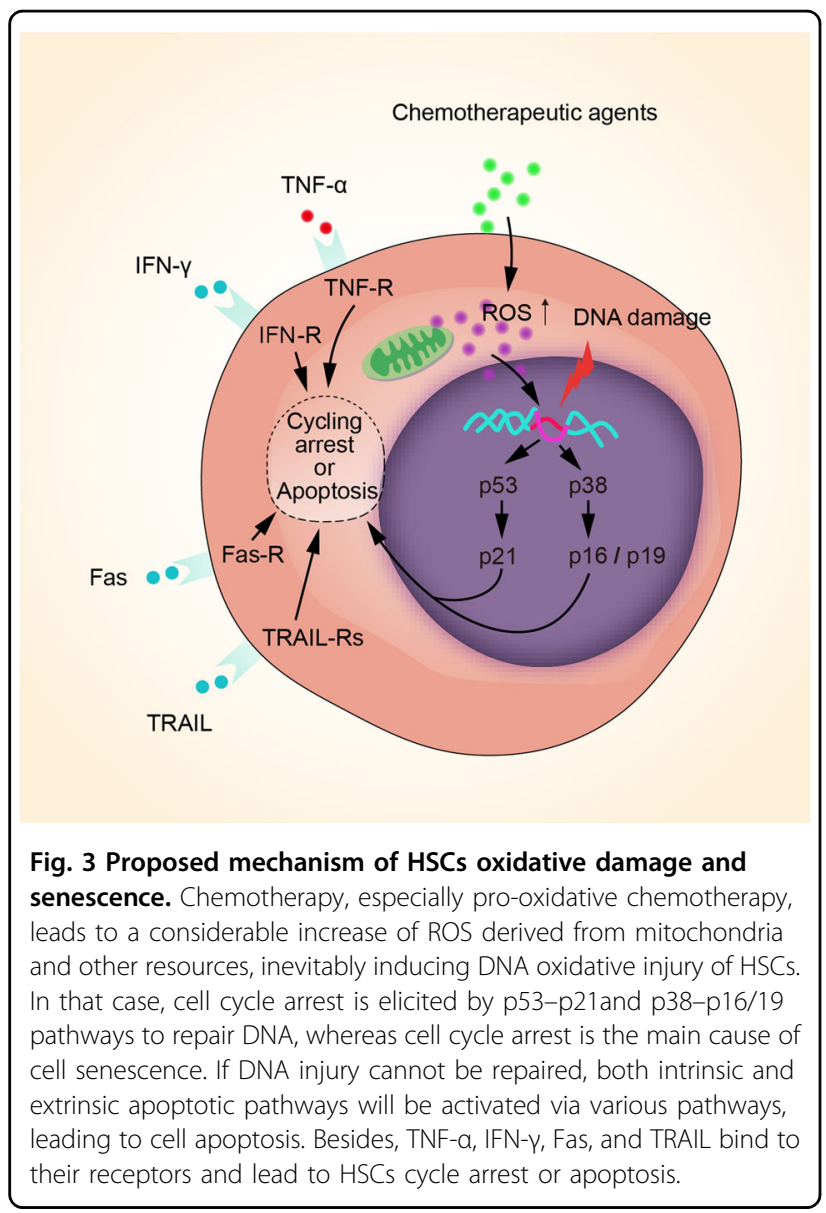

It was reported that, Isothiocyanates act by depleting GSH pools, and efficiently kill fludarabine-resistant CLL cells ${ }^{111}$ and imatinib-resistant CML cells ${ }^{112}$ selectively without attacking normal hematopoietic cells. Preclinical studies have shown that different SOD inhibitors, such as ATN$224^{113}$ and 2-methoxyestradiol (2-ME) $)^{114}$, have an antileukemia effect. It has been reported that 2-ME can target and kill leukemic cells but has no such effect on normal $\mathrm{HSCs}^{115}$.

In order to relieve the oxidative injury of conventional chemotherapeutic agents on BM hematopoietic tissues, research is underway to screen and develop natural components and biological products with antitumor effects, such as catechins, parthenolide, curcumin, and resveratrol ${ }^{116-135}$. The role of these natural antitumor drugs in inducing the apoptosis of tumor cells is also related to ROS. They can replace conventional chemotherapeutic drugs or reduce the dosage of conventional chemotherapeutic agents to a certain degree, thereby alleviating toxicity and side effects. Zhang et al. showed that catechins suppressed the proliferation of acute promyelocytic leukemia (APL) cells and elicited cell apoptosis at the micromolar concentration level, which was related to mitochondrial damage, ROS production, and caspase activation. Catechin-mediated apoptosis was also found in primary APL cells, but normal hematopoietic progenitor cells were unaffected ${ }^{121}$. According to the report by Guzman et al., parthenolide could induce the apoptosis of primary human AML cells and blast crisis CML cells by inducing ROS generation. However, parthenolide of the same concentration barely had any effects on the $\mathrm{BMHCs}^{130}$. The above studies suggest that natural antitumor drugs have broad prospects in the treatment of leukemia while protecting BM hematopoietic function. Some natural compounds that exert antileukemia activity via ROS-dependent actions are listed in Table 1. In addition, recent studies have found that many natural compounds possess potent antioxidant activity, which may protect BMHSCs from oxidative damage. It has been reported that some natural polyphenolic antioxidants, such as curcumin and quercetin, can effectively protect BMHSCs from the oxidative damage caused by prooxidative drugs without affecting the antileukemia function $^{136-138}$. It has been shown that this phenomenon is associated with the difference in ROS levels between cancer cells and healthy cells, and polyphenols may exert more pro-oxidative action in cancer cells with increased levels of $\operatorname{ROS}^{125,138}$. In recent years, many studies have shown that many alkaloids, polysaccharides, flavonoids, and saponins extracted from plants also display antitumor and antioxidant effects ${ }^{139-141}$. The antioxidative mechanism of natural antioxidants is illustrated in Fig. 4.

\section{Summary}

New treatment strategies targeting the oxidative stress status of leukemic cells and the BM microenvironment have become hot topics of research. However, many of the previous studies have limitations. First, most of them are in vitro studies, which cannot completely simulate the BM microenvironment in a physical or pathological condition. Therefore, these studies cannot truly reflect the state inside the leukemia patients' bodies. Second, leukemia is highly heterogeneous and varies across individuals, disease types, and development stages. Moreover, the cell status, oxidative stress level of cells and relevant regulation mechanisms also vary, making it very difficult to determine the appropriate ROS level for pro-oxidative treatments. Third, there are also BMSCs and HSCs in the BM microenvironment apart from the leukemic cells. Prooxidant drugs may damage HSCs and other normal cells, leading to severe BM suppression and other adverse events. In conclusion, deeper and more comprehensive studies of the redox features and regulatory mechanisms of HSCs and LSCs in the BM microenvironment are urgently needed to identify new therapeutic targets to eradicate LSCs without harming normal HSCs. This issue represents the biggest challenge in the field of leukemia treatment. 


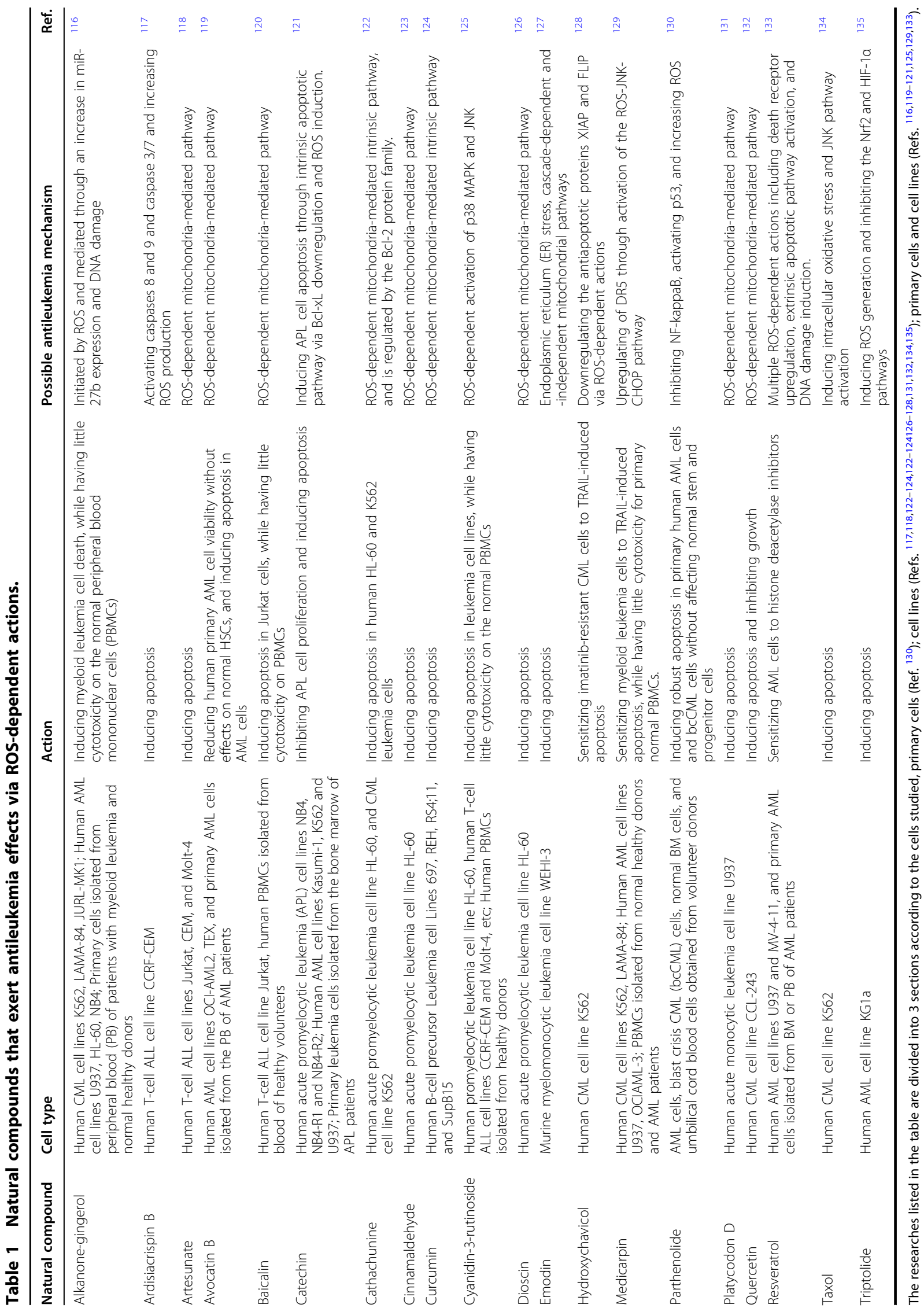




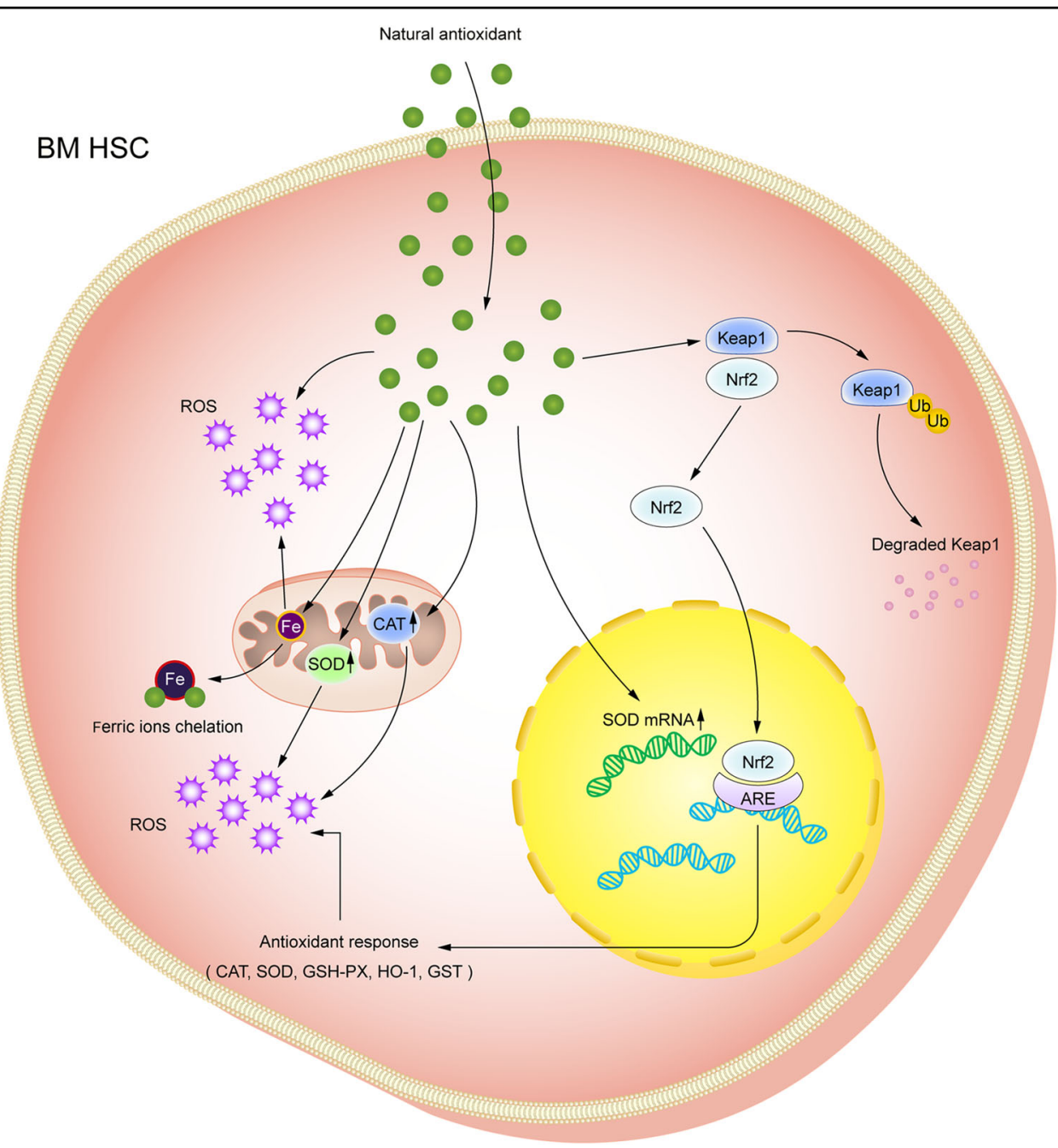

Fig. 4 Antioxidative mechanism of natural antioxidants. Natural antioxidants extracted from plants exert antioxidative effects mainly through the following pathways: 1. direct clearing of ROS; 2 . chelating ferric ions to inhibit ROS production; 3 . improving the activities of anti-oxidases such as SOD and CAT; 4. promoting SOD mRNA expressions; 5 . clearing ROS by activating the Nrf2 antioxidative pathway.

In recent years, many studies have made good use of modern biological technology. Great efforts have been devoted to developing animal models that can realistically reflect the microenvironment of leukemia patients, including transgenic models and chimeric models. The findings from these models are encouraging further studies on leukemia. With the establishment of more animal models of leukemia with high fitness, our understanding of leukemia will deepen, which will contribute to progress in leukemia studies.

\section{Acknowledgements}

This research was funded by the Public Welfare Technology Application Research Project of Zhejiang Province under grant no. 2015C37122, Zhejiang, China; and Zhejiang Provincial Natural Science Foundation of China under grant no. LY17H160003, Zhejiang, China.

\section{Competing interests}

The authors declare that they have no competing interests.

\section{Publisher's note}

Springer Nature remains neutral with regard to jurisdictional claims in published maps and institutional affiliations.

Received: 15 November 2019 Revised: 8 April 2020 Accepted: 8 April 2020 Published online: 27 April 2020

\section{References}

1. Wang, A. \& Zhong, H. Roles of the bone marrow niche in hematopoiesis, leukemogenesis, and chemotherapy resistance in acute myeloid leukemia. Hematology 23, 729-739 (2018).

2. Konopleva, M., Tabe, Y., Zeng, Z. \& Andreeff, M. Therapeutic targeting of microenvironmental interactions in leukemia: mechanisms and approaches. Drug Resist. Updates 12, 103-113 (2009). 
3. Warr, M. R., Pietras, E. M. \& Passegue, E. Mechanisms controlling hematopoietic stem cell functions during normal hematopoiesis and hematological malignancies. Wiley Interdiscip. Rev. Syst. Biol. Med. 3, 681-701 (2011).

4. Nair, R. R., Tolentino, J. H. \& Hazlehurst, L. A. Role of stat3 in transformation and drug resistance in cml. Front. Oncol. 2, 30 (2012).

5. Ushio-Fukai, M. \& Rehman, J. Redox and metabolic regulation of stem/ progenitor cells and their niche. Antioxid. Redox Signal. 21, 1587-1590 (2014).

6. Jayavelu, A. K., Moloney, J. N., Böhmer, F. D. \& Cotter, T. G. NOX-driven ROS formation in cell transformation of FLT3-ITD-positive AML. Exp. Hematol. 44 1113-1122 (2016).

7. Mi, T., Wang, Z. \& Bunting, K. D. The cooperative relationship between STAT5 and reactive oxygen species in leukemia: mechanism and therapeutic potential. Cancers (Basel) 10, pii: E359 (2018).

8. Ludin, A. et al. Reactive oxygen species regulate hematopoietic stem cell selfrenewal, migration and development, as well as their bone marrow microenvironment. Antioxid. Redox Signal. 21, 1605-1619 (2014).

9. Samimi, A., Kalantari, H., Lorestani, M. Z., Shirzad, R. \& Saki, N. Oxidative stress in normal hematopoietic stem cells and leukemia. Apmis 126, 284-294 (2018).

10. Prieto-Bermejo, R., Romo-González, M., Pérez-Fernández, A., ljurko, C. \& Hernández-Hernández, Á. Reactive oxygen species in haematopoiesis: leukaemic cells take a walk on the wild side. J. Exp. Clin. Cancer Res. 37, 125 (2018).

11. Chen, Y. F. et al. The roles of reactive oxygen species (ros) and autophagy in the survival and death of leukemia cells. Crit. Rev. Oncol. Hematol. 112, 21-30 (2017)

12. Zhou, F., Shen, Q. \& Claret, F. X. Novel roles of reactive oxygen species in the pathogenesis of acute myeloid leukemia. J. Leukoc. Biol. 94, 423-429 (2013).

13. Chen, Y. F., Wu, Z. M., Luo, X. J., Bai, S. \& Zhao, L. D. Effect of the conditional knockout of bone marrow specific RIPK3 gene on bone marrow hematopoiesis in mice. Int. J. Clin. Exp. Pathol. 11, 568-576 (2018).

14. Taniguchi Ishikawa, E. et al. Connexin-43 prevents hematopoietic stem cell senescence through transfer of reactive oxygen species to bone marrow stromal cells. Proc. Natl Acad. Sci. 109, 9071-9076 (2012).

15. Liekens, S., Schols, D. \& Hatse, S. Cxcl12-cxcr4 axis in angiogenesis, metastasis and stem cell mobilization. Curr. Pharm. Des. 16, 3903-3920 (2010).

16. Riether, C., Schurch, C. M. \& Ochsenbein, A. F. Regulation of hematopoietic and leukemic stem cells by the immune system. Cell. Death. Differ. 22 187-198 (2015).

17. Carlesso, N. \& Cardoso, A. A. Stem cell regulatory niches and their role in normal and malignant hematopoiesis. Curr. Opin. Hematol. 17, 281-286 (2010).

18. Tavor, S. et al. Cxcr4 regulates migration and development of human acute myelogenous leukemia stem cells in transplanted nod/scid mice. Cancer Res. 64, 2817-2824 (2004)

19. Gul, H., Marquez-Curtis, L. A., Jahroudi, N., Larratt, L. M. \& Janowska-Wieczorek A. Valproic acid exerts differential effects on cxcr4 expression in leukemic cells. Leuk. Res. 34, 235-242 (2010)

20. Zhang, H., Li, H., Xi, H. S. \& Li, S. HIF1a is required for survival maintenance of chronic myeloid leukemia stem cells. Blood 119, 2595-2607 (2012).

21. Schepers, K., Campbell, T. B. \& Passegue, E. Normal and leukemic stem cell niches: insights and therapeutic opportunities. Cell Stem Cell 16, 254-267 (2015).

22. Morikawa, T. \& Takubo, K. Hypoxia regulates the hematopoietic stem cell niche. Pflug. Arch. 468, 13-22 (2016)

23. Goto, M. et al. Adaptation of leukemia cells to hypoxic condition through switching the energy metabolism or avoiding the oxidative stress. BMC Cancer 14, 76 (2014).

24. Tadokoro, H., Umezu, T., Ohyashiki, K., Hirano, T. \& Ohyashiki, J. H. Exosomes derived from hypoxic leukemia cells enhance tube formation in endothelial cells. J. Biol. Chem. 288, 34343-34351 (2013).

25. Zhe, N. et al. HIF-1a inhibition by 2-methoxyestradiol induces cell death via activation of the mitochondrial apoptotic pathway in acute myeloid leukemia. Cancer Biol. Ther. 17, 625-634 (2016).

26. Zeng, D. et al. Ginsenoside Rg3 inhibits HIF-1a and VEGF expression in patient with acute leukemia via inhibiting the activation of PI3K/Akt and ERK1/2 pathways. Int. J. Clin. Exp. Pathol. 7, 2172-2178 (2014).

27. $\mathrm{Xu}, \mathrm{Q}$. et al. The effect of ruxolitinib on the expression of vegf and hif-1alpha in leukemia hel cells. J. Sichuan Univ. (Med. Sci. Ed.) 47, 669-673 (2016).

28. Chen, F. et al. Triptolide, a chinese herbal extract, enhances drug sensitivity of resistant myeloid leukemia cell lines through downregulation of HIF-1a and Nrf2. Pharmacogenomics 14, 1305-1317 (2013).
29. Shirzad, R. et al. Signaling and molecular basis of bone marrow niche angiogenesis in leukemia. Clin. Transl. Oncol. 18, 957-971 (2016).

30. Testa, U., Labbaye, C., Castelli, G. \& Pelosi, E. Oxidative stress and hypoxia in normal and leukemic stem cells. Exp. Hematol. 44, 540-560 (2016).

31. Lagadinou, E. D. et al. BCl-2 inhibition targets oxidative phosphorylation and selectively eradicates quiescent human leukemia stem cells. Cell Stem Cell 12, 329-341 (2013).

32. Marlein, C. R. et al. Nadph oxidase-2 derived superoxide drives mitochondrial transfer from bone marrow stromal cells to leukemic blasts. Blood $\mathbf{1 3 0}$ 1649-1660 (2017).

33. Kumar, B. et al. Acute myeloid leukemia transforms the bone marrow niche into a leukemia-permissive microenvironment through exosome secretion. Leukemia 32, 575-587 (2018).

34. Huan, J. et al. Coordinate regulation of residual bone marrow function by paracrine trafficking of aml exosomes. Leukemia 29, 2285-2295 (2015).

35. Asada, N. \& Katayama, Y. Regulation of hematopoiesis in endosteal microenvironments. Int. J. Hematol. 99, 679-684 (2014).

36. Baba, T. et al. MIP-1a/CCL3-mediated maintenance of leukemia-initiating cells in the initiation process of chronic myeloid leukemia. J. Exp. Med. 210, 2661-2673 (2013)

37. Frisch, B. J. et al. Functional inhibition of osteoblastic cells in an in vivo mouse model of myeloid leukemia. Blood 119, 540-550 (2012).

38. Schepers, K. et al. Myeloproliferative neoplasia remodels the endosteal bone marrow niche into a self-reinforcing leukemic niche. Cell Stem Cell 13, 285-299 (2013).

39. Diehn, $\mathbf{M}$. et al. Association of reactive oxygen species levels and radioresistance in cancer stem cells. Nature 458, 780-783 (2009).

40. Herault, $\mathrm{O}$. et al. A role for GPx3 in activity of normal and leukemia stem cells. J. Exp. Med. 209, 895-901 (2012).

41. Paraguassu-Braga, F. H., Borojevic, R., Bouzas, L. F., Barcinski, M. A. \& Bonomo, A. Bone marrow stroma inhibits proliferation and apoptosis in leukemic cells through gap junction-mediated cell communication. Cell Death Differ. 10 1101-1108 (2003).

42. Ichihara, E., Kaneda, K, Saito, Y., Yamakawa, N. \& Morishita, K. Angiopoietin1 contributes to the maintenance of cell quiescence in evi1high leukemia cells. Biochem. Biophys. Res. Commun. 416, 239-245 (2011).

43. Hole, P. S. et al. Overproduction of nox-derived ros in aml promotes proliferation and is associated with defective oxidative stress signaling. Blood 122, 3322-3330 (2013)

44. Singh, M. M. et al. Inhibition of the nadph oxidase regulates heme oxygenase 1 expression in chronic myeloid leukemia. Cancer $\mathbf{1 1 8}$ 3433-3445 (2012)

45. Dong, J. M., Zhao, S. G., Huang, G. Y. \& Liu, Q. Nadph oxidase-mediated generation of reactive oxygen species is critically required for survival of undifferentiated human promyelocytic leukemia cell line HL-60. Free Radic. Res. 38, 629-637 (2004).

46. Irwin, M. E., Rivera-Del Valle, N. \& Chandra, J. Redox control of leukemia: from molecular mechanisms to therapeutic opportunities. Antioxid. Redox Signal. 18, 1349-1383 (2013).

47. Romeo, M. et al. The human t-cell leukemia virus type-1 p30(ii) protein activates p53 and induces the tigar and suppresses oncogene-induced oxidative stress during viral carcinogenesis. Virology 518, 103-115 (2018).

48. Tahir, I. M., Iqbal, T., Jamil, A. \& Saqib, M. Association of bcl-2 with oxidative stress and total antioxidant status in pediatric acute lymphoblastic leukemia. J. Biol. Regul. Homeost. Agents 31, 1023-1027 (2017).

49. Rasool, M. et al. Assessment of circulating biochemical markers and antioxidative status in acute lymphoblastic leukemia (all) and acute myeloid leukemia (aml) patients. Saudi J. Biol. Sci. 22, 106-111 (2015).

50. Gaman, A. M., Buga, A. M., Gaman, M. A. \& Popa-Wagner, A. The role of oxidative stress and the effects of antioxidants on the incidence of infectious complications of chronic lymphocytic leukemia. Oxid. Med. Cell Longev. 2014 158135 (2014)

51. Battisti, V. et al. Measurement of oxidative stress and antioxidant status in acute lymphoblastic leukemia patients. Clin. Biochem. 41, 511-518 (2008).

52. Bourgeais, J. et al. Oncogenic STAT5 signaling promotes oxidative stress in chronic myeloid leukemia cells by repressing antioxidant defenses. Oncotarget 8, 41876-41889 (2017).

53. Kuntz, E. M. et al. Targeting mitochondrial oxidative phosphorylation eradicates therapy-resistant chronic myeloid leukemia stem cells. Nat. Med $\mathbf{2 3}$ 1234-1240 (2017). 
54. Sriskanthadevan, S. et al. AML cells have low spare reserve capacity in their respiratory chain that renders them susceptible to oxidative metabolic stress. Blood 125, 2120-2130 (2015).

55. Bolton-Gillespie, E. Genomic instability may originate from imatinib-refractory chronic myeloid leukemia stem cells. Blood 121, 4175-4183 (2013).

56. $\mathrm{Fu}, \mathrm{X}$. et al. Targeting mitochondrial respiration selectively sensitizes pediatric acute lymphoblastic leukemia cell lines and patient samples to standard chemotherapy. Am. J. Cancer Res. 7, 2395-2405 (2017).

57. Jitschin, R. et al. Mitochondrial metabolism contributes to oxidative stress and reveals therapeutic targets in chronic lymphocytic leukemia. Blood 123 2663-2672 (2014).

58. Guachalla, L. M. \& Rudolph, K. L. Ros induced DNA damage and checkpoint responses: influences on aging? Cell Cycle 9, 4058-4060 (2010).

59. Groninger, E., Meeuwsen-De Boer, G. J., De Graaf, S. S. N., Kamps, W. A. \& De Bont, E. S. J. M. Vincristine induced apoptosis in acute lymphoblastic leukaemia cells: a mitochondrial controlled pathway regulated by reactive oxygen species? Int. J. Oncol. 21, 1339-1345 (2002).

60. Mizutani, H., Tada-Oikawa, S., Hiraku, Y., Kojima, M. \& Kawanishi, S. Mechanism of apoptosis induced by doxorubicin through the generation of hydrogen peroxide. Life Sci. 76, 1439-1453 (2005).

61. Romano, M. F. et al. Enhancement of cytosine arabinoside-induced apoptosis in human myeloblastic leukemia cells by NF-kappa B/Rel- specific decoy oligodeoxynucleotides. Gene. Ther. 7, 1234-1237 (2000).

62. Kanno, S. et al. Susceptibility to cytosine arabinoside (Ara-C)-induced cytotoxicity in human leukemia cell lines. Toxicol. Lett. 152, 149-158 (2004).

63. Jing, B. et al. Vorinostat and quinacrine have synergistic effects in t-cell acute lymphoblastic leukemia through reactive oxygen species increase and mitophagy inhibition. Cell Death Dis. 9, 589 (2018).

64. Silic-Benussi, M. et al. Selective killing of human t-all cells: an integrated approach targeting redox homeostasis and the oma1/opa1 axis. Cell Death Dis. 9, 822 (2018)

65. Kelly, B., Tannahill, G. M., Murphy, M. P. \& O'Neill, L. A. J. Metformin inhibits the production of reactive oxygen species from $\mathrm{NADH}$ : ubiquinone oxidoreductase to limit induction of interleukin-1 $\beta$ (IL-1 $\beta$ ) and boosts interleukin-10 (IL-10) in lipopolysaccharide (LPS)-activated macrophages. J. Biol. Chem. 290 20348-20359 (2015).

66. Le, S. B. et al. Inhibition of mitochondrial respiration as a source of adaphostin-induced reactive oxygen species and cytotoxicity. J. Biol. Chem 282, 8860-8872 (2007)

67. Chandra, J. et al. Adaphostin-induced oxidative stress overcomes bcr/ab mutation-dependent and -independent imatinib resistance. Blood $\mathbf{1 0 7}$ 2501-2506 (2006).

68. Huang, $X$. et al. Bypassing drug resistance by triggering necroptosis: recent advances in mechanisms and its therapeutic exploitation in leukemia. J. Exp. Clin. Cancer Res. 37, 310 (2018).

69. Chen, Y. F. et al. TNF-a-induced programmed cell death in the pathogenesis of acquired aplastic anemia. Expert. Rev. Hematol. 8, 515-526 (2015).

70. Shehata, M. et al. Reconstitution of PTEN activity by CK2 inhibitors and interference with the PI3-K/Akt cascade counteract the antiapoptotic effect of human stromal cells in chronic lymphocytic leukemia. Blood $\mathbf{1 1 6}$ 2513-2521 (2010).

71. Iwamoto, S., Mihara, K., Downing, J. R., Pui, C. H. \& Campana, D. Mesenchymal cells regulate the response of acute lymphoblastic leukemia cells to asparaginase. J. Clin. Invest. 117, 1049-1057 (2007)

72. Roodhart, J. M. et al. Mesenchymal stem cells induce resistance to chemotherapy through the release of platinum-induced fatty acids. Cancer Cell 20, 370-383 (2011)

73. Zhang, W. et al. Stromal control of cystine metabolism promotes cancer cel survival in chronic lymphocytic leukaemia. Nat. Cell Biol. 14, 276-286 (2012).

74. Ding, L. et al. Targeting the autophagy in bone marrow stromal cells overcomes resistance to vorinostat in chronic lymphocytic leukemia. Onco. Targets Ther. 11, 5151-5170 (2018).

75. Sheng, X. et al. Adipocytes cause leukemia cell resistance to daunorubicin via oxidative stress response. Oncotarget 7, 73147-73159 (2016).

76. Cort, A., Ozben, T., Saso, L., De Luca, C. \& Korkina, L. Redox control of multidrug resistance and its possible modulation by antioxidants. Oxid. Med. Cell Longev. 2016, 4251912 (2016)

77. Schoeneberger, H., Belz, K., Schenk, B. \& Fulda, S. Impairment of antioxidant defense via glutathione depletion sensitizes acute lymphoblastic leukemia cells for Smac mimetic-induced cell death. Oncogene 34, 4032-4043 (2015).
78. Haß, C., Belz, K., Schoeneberger, H. \& Fulda, S. Sensitization of acute lymphoblastic leukemia cells for LCL161-induced cell death by targeting redox homeostasis. Biochem. Pharmacol. 105, 14-22 (2016).

79. Tan, Y. et al. Thioredoxin-1 inhibitor PX-12 induces human acute myeloid leukemia cell apoptosis and enhances the sensitivity of cells to arsenic trioxide. Int. J. Clin. Exp. Pathol. 7, 4765-4773 (2014)

80. Liu, J. J. et al. Inhibition of thioredoxin reductase by auranofin induces apoptosis in adriamycin-resistant human K562 chronic myeloid leukemia cells. Pharmazie 66, 440-444 (2011).

81. Fiskus, W. et al. Auranofin induces lethal oxidative and endoplasmic reticulum stress and exerts potent preclinical activity against chronic lymphocytic leukemia. Cancer Res. 74, 2520-2532 (2014).

82. Jang, J. S., Piao, S., Cha, Y. N. \& Kim, C. Taurine chloramine activates Nrf2 increases $\mathrm{HO}-1$ expression and protects cells from death caused by hydrogen peroxide. J. Clin. Biochem. Nutr. 45, 37-43 (2009).

83. Zhou, S., Sun, W., Zhang, Z. \& Zheng, Y. The role of Nif2-mediated pathway in cardiac remodeling and heart failure. Oxid. Med. Cell Longev. 2014, 260429 (2014).

84. $\mathrm{Xu}, \mathrm{L}$. et al. Inhibition of the Nrf2-TrxR axis sensitizes the drug-resistant chronic myelogenous leukemia cell line K562/G01 to imatinib treatments. Biomed. Res. Int. 2019, 6502793 (2019).

85. Liu, J. et al. Stromal cell-mediated mitochondrial redox adaptation regulates drug resistance in childhood acute lymphoblastic leukemia. Oncotarget $\mathbf{6}$ 43048-43064 (2015)

86. Hosseini, M. et al. Targeting myeloperoxidase disrupts mitochondrial redox balance and overcomes cytarabine resistance in human acute myeloid leukemia. Cancer Res. 79, 5191-5203 (2019).

87. Li Volti, G. et al. The heme oxygenase system in hematological malignancies. Antioxid. Redox Signal. 27, 363-377 (2017).

88. Saito, Y., Chapple, R. H., Lin, A., Kitano, A. \& Nakada, D. AMPK protects leukemia-initiating cells in myeloid leukemias from metabolic stress in the bone marrow. Cell Stem Cell 17, 585-596 (2015).

89. Casetti, L. et al. Differential contributions of STAT5A and STAT5B to stress protection and tyrosine kinase inhibitor resistance of chronic myeloid leukemia stem/progenitor cells. Cancer Res. 73, 2052-2058 (2013).

90. Mesbahi, Y. et al. Blockade of JAK2/STAT3 intensifies the anti-tumor activity of arsenic trioxide in acute myeloid leukemia cells: novel synergistic mechanism via the mediation of reactive oxygen species. Eur. J. Pharm. 834, 65-76 (2018).

91. Kelley, E. E., Wagner, B. A., Buettner, G. R. \& Burns, C. P. Nitric oxide inhibits iron-induced lipid peroxidation in HL-60 cells. Arch. Biochem. Biophys. 370 97-104 (1999).

92. Gorbunov, N. V. et al. Nitric oxide prevents oxidative damage produced by tert-butyl hydroperoxide in erythroleukemia cells via nitrosylation of heme and non-heme iron. Electron paramagnetic resonance evidence. J. Biol. Chem. 272, 12328-12341 (1997).

93. Umansky, V. et al. Inhibition of nitric-oxide-mediated apoptosis in Jurkat leukemia cells despite cytochrome c release. Exp. Cell Res. 265, 274-282 (2001)

94. Rubbo, H. et al. Nitric oxide regulation of superoxide and peroxynitritedependent lipid peroxidation. Formation of novel nitrogen-containing oxidized lipid derivatives. J. Biol. Chem. 269, 26066-26075 (1994).

95. Takahashi, H. et al. Autophagy is required for cell survival under Iasparaginase-induced metabolic stress in acute lymphoblastic leukemia cells. Oncogene 36, 4267-4276 (2017)

96. Folkerts, H. et al. Inhibition of autophagy as a treatment strategy for p53 wildtype acute myeloid leukemia. Cell Death Dis. 8, e2927 (2017).

97. $\mathrm{Wu}, \mathrm{X}$. et al. Role of beclin-1-mediated autophagy in the survival of pediatric leukemia cells. Cell Physiol. Biochem. 39, 1827-1836 (2016).

98. Tang, C. et al. Chemotherapy-induced niche perturbs hematopoietic reconstitution in b-cell acute lymphoblastic leukemia. J. Exp. Clin. Cancer Res. 37, 204 (2018)

99. Yahata, T. et al. Accumulation of oxidative DNA damage restricts the selfrenewal capacity of human hematopoietic stem cells. Blood 118, 2941-2950 (2011)

100. Singh, A. K., Althoff, M. J. \& Cancelas, J. A. Signaling pathways regulating hematopoietic stem cell and progenitor aging. Curr. Stem. Cell Rep. $\mathbf{4}$ 166-181 (2018).

101. Mortensen, M., Watson, A. S. \& Simon, A. K. Lack of autophagy in the hematopoietic system leads to loss of hematopoietic stem cell function and dysregulated myeloid proliferation. Autophagy 7, 1069-1070 (2011). 
102. Ho, T. T. Autophagy maintains the metabolism and function of young and old stem cells. Nature 543, 205-210 (2017).

103. Garg, A. D. et al. ROS-induced autophagy in cancer cells assists in evasion from determinants of immunogenic cell death. Autophagy 9, 1292-1307 (2013)

104. Poillet-Perez, L., Despouy, G., Delage-Mourroux, R. \& Boyer-Guittaut, M. Interplay between ROS and autophagy in cancer cells, from tumor initiation to cancer therapy. Redox Biol. 4, 184-192 (2015).

105. Baquero, P., Dawson, A. \& Helgason, G. V. Autophagy and mitochondrial metabolism: insights into their role and therapeutic potential in chronic myeloid leukaemia. FEBS J. 286, 1271-1283 (2019).

106. Evangelisti, C. et al. Autophagy in acute leukemias: a double-edged sword with important therapeutic implications. Biochim. Biophys. Acta 1853, 14-26 (2015)

107. Puissant, A. et al. Resveratrol promotes autophagic cell death in chronic myelogenous leukemia cells via JNK-mediated p62/SQSTM1 expression and AMPK activation. Cancer Res. 70, 1042-1052 (2010).

108. Townsend, D. M., Findlay, V. L. \& Tew, K. D. Glutathione S-transferases as regulators of kinase pathways and anticancer drug targets. Methods enzymol. 401, 287-307 (2005)

109. Miyazaki, T. et al. Expression of heme oxygenase-1 in human leukemic cells and its regulation by transcriptional repressor Bach1. Cancer Sci. 101 1409-1416 (2010).

110. Udensi, U. K. \& Tchounwou, P. B. Dual effect of oxidative stress on leukemia cancer induction and treatment. J. Exp. Clin. Cancer Res. 33, 106 (2014).

111. Trachootham, D. et al. Effective elimination of fludarabine-resistant CLL cells by PEITC through a redox-mediated mechanism. Blood 112, 1912-1922 (2008).

112. Zhang, $H$. et al. Effective killing of Gleevec-resistant CML cells with T315I mutation by a natural compound PEITC through redox-mediated mechanism. Leukemia 22, 1191-1199 (2008).

113. Lee, K. et al. The copper chelator ATN-224 induces peroxynitrite-dependent cell death in hematological malignancies. Free Radic. Biol. Med. 60, 157-167 (2013)

114. Gao, N., Rahmani, M., Dent, P. \& Grant, S. 2-methoxyestradiol-induced apoptosis in human leukemia cells proceeds through a reactive oxygen species and akt-dependent process. Oncogene 24, 3797-3809 (2005).

115. Gerby, B. et al. High-throughput screening in niche-based assay identifies compounds to target preleukemic stem cells. J. Clin. Invest 126, 4569-4584 (2016).

116. Rastogi, N. et al. (6)-Gingerol induced myeloid leukemia cell death is initiated by reactive oxygen species and activation of miR-27b expression. Free Radic Biol. Med. 68, 288-301 (2014).

117. Mbaveng, A. T. et al. A naturally occuring triterpene saponin ardisiacrispin B displayed cytotoxic effects in multi-factorial drug resistant cancer cells via ferroptotic and apoptotic cell death. Phytomedicine $\mathbf{4 3}$ 78-85 (2018)

118. Efferth, T., Giaisi, M., Merling, A., Krammer, P. H. \& Li-Weber, M. Artesunate induces ros-mediated apoptosis in doxorubicin-resistant t leukemia cells. PLOS ONE 2, e693 (2007).

119. Lee, E. A. et al. Targeting mitochondria with avocatin b induces selective leukemia cell death. Cancer Res. 75, 2478-2488 (2015).

120. Ueda, S. et al. Baicalin induces apoptosis via mitochondrial pathway as prooxidant. Mol. Immunol. 38, 781-791 (2002).

121. Zhang, L. et al. Catechins induced acute promyelocytic leukemia cell apoptosis and triggered pml-rara oncoprotein degradation. J. Hematol. Oncol. 7, 75 (2014)

122. Wang, X. D. et al. Induction of apoptosis in human leukemia cells through an intrinsic pathway by cathachunine, a unique alkaloid isolated from Catharanthus roseus. Phytomedicine 23, 641-653 (2016).
123. Ka, $\mathrm{H}$. et al. Cinnamaldehyde induces apoptosis by ros-mediated mitochondrial permeability transition in human promyelocytic leukemia HL-60 cells. Cancer Lett. 196, 143-152 (2003).

124. Kuttikrishnan, S. et al. Curcumin induces apoptotic cell death via inhibition of $\mathrm{PI3-kinase/AKT} \mathrm{pathway} \mathrm{in} \mathrm{B-precursor} \mathrm{acute} \mathrm{lymphoblastic} \mathrm{leukemia.} \mathrm{Front.}$ Oncol. 9, 484 (2019).

125. Feng, R. et al. Cyanidin-3-rutinoside, a natural polyphenol antioxidant, selectively kills leukemic cells by induction of oxidative stress. J. Biol. Chem. 282, 13468-13476 (2007)

126. Wang, Y., Che, C. M., Chiu, J. F. \& He, Q. F. Dioscin (saponin)-induced generation of reactive oxygen species through mitochondria dysfunction: a proteomic-based study. J. Proteome Res. 6, 4703-4710 (2007).

127. Chang, Y. C. et al. Emodin induces apoptotic death in murine myelomonocytic leukemia wehi-3 cells in vitro and enhances phagocytosis in leukemia mice in vivo. Evid. Based Complement. Altern. Med 2011, 523596 (2011)

128. Paul, T., Banerjee, A., Reddy, S. V. B., Mahato, S. K. \& Biswas, N. Hydroxychavicol sensitizes imatinib-resistant chronic myelogenous leukemia cells to TRAlLinduced apoptosis by ROS-mediated IAP downregulation. Anticancer Drugs 30, 167-178 (2019)

129. Trivedi, R., Maurya, R. \& Mishra, D. P. Medicarpin, a legume phytoalexin sensitizes myeloid leukemia cells to trail-induced apoptosis through the induction of DR5 and activation of the ROS-JNK-CHOP pathway. Cell Death Dis. 5, e1465 (2014).

130. Guzman, M. L. et al. The sesquiterpene lactone parthenolide induces apoptosis of human acute myelogenous leukemia stem and progenitor cells. Blood 105, 4163-4169 (2005).

131. Shin, D. Y. et al. Implication of intracellular ROS formation, caspase-3 activation and Egr-1 induction in platycodon D-induced apoptosis of U937 human leukemia cells. Biomed. Pharmacother. 63, 86-94 (2009).

132. Mutlu Altundağ, E., Yılmaz, A. M., Koçtürk, S., Taga, Y. \& Yalçın, A. S. Synergistic induction of apoptosis by quercetin and curcumin in chronic myeloid leukemia(K562) cells. Nutr. Cancer 70, 97-108 (2018).

133. Yaseen, $\mathrm{A}$. et al. Resveratrol sensitizes acute myelogenous leukemia cells to histone deacetylase inhibitors through reactive oxygen species-mediated activation of the extrinsic apoptotic pathway. Mol. Pharmacol. 82, 1030-1041 (2012).

134. Liu, Y. et al. Low-dose triptolide in combination with idarubicin induces apoptosis in AML leukemic stem-likeKG1a cell line by modulation of the intrinsic and extrinsic factors. Cell Death Dis. 4, e948 (2013).

135. Meshkini, A. \& Yazdanparast, R. Involvement of oxidative stress in taxolinduced apoptosis in chronic myelogenous leukemia K562 cells. Exp. Toxicol. Pathol. 64, 357-365 (2012)

136. Papiez, M. A. \& Krzyściak, W. The dual effect of curcumin on etoposide action in leukemic and healthy bone marrow cells of rats with acute myeloid leukemia. Folia Med. Cracov. 54, 71-79 (2014).

137. Papiez, M. A. The effect of quercetin on oxidative DNA damage and myelosuppression induced by etoposide in bone marrow cells of rats. Acto Biochim. Pol. 61, 7-11 (2014).

138. Papiez, M. A. The influence of curcumin and (-)-epicatechin on the genotoxicity and myelosuppression induced by etoposide in bone marrow cells of male rats. Drug Chem. Toxicol. 36, 93-101 (2013).

139. Hu, J. et al. Ultrasonic extraction, antioxidant and anticancer activities of nove polysaccharides from Chuanxiong rhizome. Int. J. Biol. Macromol. 85, 277-284 (2016).

140. Zhao, J. et al. Evaluation on anti-inflammatory, analgesic, antitumor, and antioxidant potential of total saponins from nigella glandulifera seeds. Evid. Based Complement. Altern. Med. 2013, 827230 (2013).

141. Bokemeyer, C. et al. Silibinin protects against cisplatin-induced nephrotoxicity without compromising cisplatin or ifosfamide anti-tumour activity. $\mathrm{Br}$. Cancer 74, 2036-2041 (1996). 